Résumés des conférences et travaux

\title{
Dialectologie du grec ancien
}

\section{Sophie Minon}

\section{OpenEdition \\ Journals}

Édition électronique

URL : https://journals.openedition.org/ashp/2280

DOI : $10.4000 /$ ashp. 2280

ISSN : 1969-6310

Éditeur

Publications de l'École Pratique des Hautes Études

Édition imprimée

Date de publication : 1 septembre 2018

Pagination : 84-92

ISSN : 0766-0677

Référence électronique

Sophie Minon, «Dialectologie du grec ancien », Annuaire de l'École pratique des hautes études (EPHE), Section des sciences historiques et philologiques [En ligne], 149 | 2018, mis en ligne le 09 juillet 2018, consulté le 03 août 2021. URL : http://journals.openedition.org/ashp/2280 ; DOI : https://doi.org/ 10.4000/ashp. 2280 


\title{
DIALECTOLOGIE DU GREC ANCIEN
}

\author{
Directeur d'études : $\mathrm{M}^{\mathrm{me}}$ Sophie MinoN
}

Programme de l'année 2016-2017 : I. Inscriptions dialectales du Péloponnèse et de Grèce septentrionale. Nouveautés dialectologiques. - II. Prose administrative, prose scientifique et prose d'art, de l'attique classique aux koinai de l'époque hellénistique et impériale : langue et style. - III. Noms de personnes et histoire des mots : analyse linguistique des anthroponymes grecs antiques en ligne, projet LGPN-Ling, en préparation du nouveau Bechtel (Die historischen Personennamen des Griechischen bis zur Kaiserzeit, 1917).

Reprise du séminaire le mardi 8 novembre 2016. Présents 15 auditeurs, dont 4 nouveaux : Vincent Bernard-La Foucrière (ENS Ulm), Louise Benkimoun (diplôme EPHE) et deux étudiantes Erasmus présentes jusqu'en janvier, Matilde Garré, université du Sacré-Cœur de Milan, et Francesca Iacono, université de Padoue, l'une et l'autre en master 2 de philologie classique.

Présentation du programme de l'année et des événements particuliers, tels le séminaire animé par Laurent Dubois (directeur d'études émérite) et Madeleine Jost (professeur émérite Paris-Ouest Nanterre) le 10 janvier 2017 pour présenter la nouvelle inscription archaïque arcadienne, publiée dans Kernos 2016 par nos collègues J. Clackson (université de Cambridge) et Y.-M. Carbon (université de Liège). Annonce des quatre conférences du professeur José Luis Garcia Ramon la semaine du 31 janvier 2017 au 3 février, à notre séminaire, à ceux de G.-J. Pinault (EPHE), de C. Le Feuvre (Paris-Sorbonne) et de D. Petit (ENS, EPHE). Le professeur Catherine Dobias-Lalou (émérite, université de Bourgogne) est invitée à parler au séminaire le 21 mars. Le professeur Jeremy Rau est invité par G.-J. Pinault et moi-même au mois d'avril.

\section{Prose scientifique. De la dialectalisation littéraire comme marque d'école}

Étude des fragments transmis par Stobée du traité pseudo-pythagoricien Пвpì vó Constantin Macris (CNRS) sur les Pseudo-Pythagorica, labex Hastec.

Introduction sur la dialectologie littéraire et problématisation : étude du mélange dialectal. Interdialectalisation corollaire de l'intertextualité. Constitution de langues poétiques dialectalement mêlées et de proses dialectalisées. Historique de la naissance des langues correspondants aux différents genres littéraires. Exemple de la dialectalisation des mimiambes du poète alexandrin Hérondas. Prépondérance de la question du genre littéraire sur celle du dialecte maternel de l'auteur.

Prose scientifique et philosophique ionienne mais pseudo-pythagorica rédigés en dorien, comme la plupart des écrits pseudo-archytéens. La dorisation, puis koinaisation, plutôt que koinéisation littéraire a-t-elle pu être motivée par la volonté de démarquer les écrits de l'école pythagoricienne de la production philosophique des écoles antérieures, école scientifique de Milet, autour du mathématicien Thalès et 
de pré-socratiques comme Xénophane de Colophon et Mélissos de Samos, et par la suite, pour les descendants d'Archytas ou de Philolaos, de se démarquer de la production en dialecte attique de l'Académie et du Lycée?

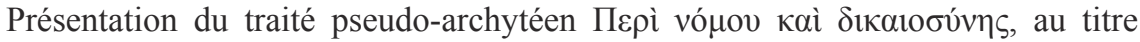
ionisé, dont l'unique source est le compilateur $\mathrm{du} \mathrm{V}^{\mathrm{e}}$ siècle de notre ère, Jean de Stobée. Questions de la datation (ca 300 a.C. au plus tôt, si l'on suit P. Horky, coéditeur avec Monte Johnson d'une réédition des fragments du traité à paraître à Oxford en 2018, PDF disponible en ligne sur son site Academia.edu), du lieu de rédaction et de la nature du mélange linguistique qu'illustrent ses fragments. Difficulté de la reconstitution d'une tradition qui couvre un empan chronologique qui s'étend de ca 300 a.C. au plus tôt jusqu'à l'époque des manuscrits de Stobée, datés du Moyen Âge (entre XII ${ }^{\mathrm{e}}$ et $\mathrm{XV}^{\mathrm{e}}$ siècle, cf. M. Curnis, L'Antologia di Giovanni Stobeo: una biblioteca antica dai manoscritti alle stampe, Alexandrie, 2008).

La comparaison avec le Fr. 1 de l'Harmonikos considéré comme le seul fragment authentique d'Archytas (428-347 a.C. environ) montre que la structure linguistique du traité pseudépigraphique n'en est pas si différente. Cela rejoint la thèse de Thesleff (An Introduction to the Pythagorean writings of the Hellenistic Period, Abo, 1961), de l'homogénéité de la tradition pythagoricienne dorienne, illustration, selon lui, d'une koinè qu'il caractérise comme italiote.

Analyse linguistique du fragment 1 de l'Harmonikos et analyse synthétique de

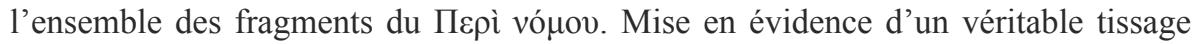
dialectal, à l'artificialité caractéristique des pseudo-pythagorica dorica, sans commune mesure avec le mixage caractéristique des langues poétiques, épique ou lyrique, comme celle de Pindare, chez qui une couleur dialectale est dominante, la même coloration dorienne en l'occurrence, mais émaillée, chez ce poète, de traits empruntés à un autre dialecte, en l'occurrence des éolismes, non homériques, mais inspirés de la langue de la lyrique lesbienne : ce sont les musiciens lesbiens qui auraient stimulé le développement de la lyrique chorale; leur prestige est rendu manifeste par la présence d'une forme lesbienne comme $\mu$ oĩ $\sigma \alpha$ dans un fragment de lyrique chorale dorienne du poète de la $2^{\text {nde }}$ moitié du VIII ${ }^{\mathrm{e}}$ s., Eumèlos de Corinthe, cf. C. J. Ruijgh, « Mycenaean and Homeric Language », chap. 16, dans Y. Duhoux, A. Morpurgo Davies (éd.), A Companion to Linear B. Mycenaean Greek Texts and their World, 2, 2011, 259.

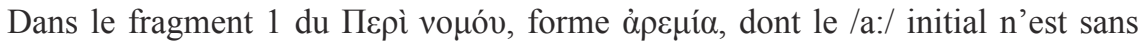
doute pas étymologique; la difficulté de l'étymologie laisse soupçonner que les Anciens ne la connaissaient pas davantage : dans ces conditions, cette forme serait moins un hyperdorisme ou hyperkoinaïsme que l'indice de la polarisation, c'est-àdire d'une surenchère dans la dialectalisation, cf. R. Hodot « Dialecte et koinè ", Lalies 9, 1990, 60. Autres exemples d'alphas employés pour noter d'anciens / $\varepsilon$ :/ étymologiques. Une analyse en contexte permet de distinguer entre dorismes anciens, dorismes par polarisation, hyperdorismes dénotant des récritures postérieures à la phase présumée originelle de rédaction du traité, probablement la haute époque hellénistique : il serait ainsi possible de distinguer entre interventions ponctuelles datables au plus tôt de l'époque hellénistique tardive, entre le $\mathrm{I}^{\mathrm{er}}$ siècle avant et le $\mathrm{I}^{\mathrm{er}}$ siècle de notre ère - ce qui correspondrait au néopythagorisme, c'est-à-dire à une période de travail intense sur les textes de la doctrine -, sinon des premiers siècles de l'Empire, 
et celles qui sont à mettre au compte de la tradition manuscrite de Stobée. Même pratique du code-switching dans ce traité que dans le fragment de l'Harmonikos, mais les illustrations en sont plus nombreuses du fait de fréquentes citations de Platon et d'Aristote ou de l'intégration de mots de leur lexique dans leur forme d'origine.

G. Genevrois présente un excursus crétois dont le point de départ est la forme

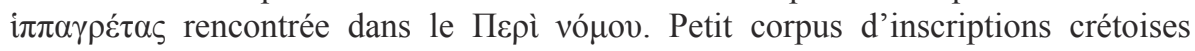
d'époque archaïque, dont la plus ancienne, à Dréros, remonte au VII ${ }^{\mathrm{e}} \mathrm{s} . a$.C. Réflexions

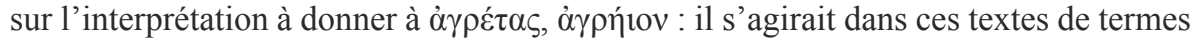
en rapport avec la notion de "saisir, collecter » plus précise que celle de « rassem-

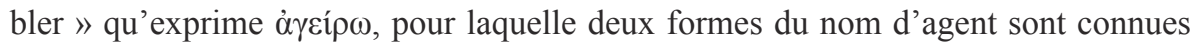

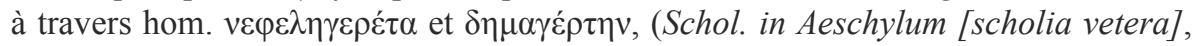
572e, 1. 1), cf. le parfait tardif $\dot{\alpha} \gamma \eta \dot{\gamma \varepsilon \rho \tau \alpha 1, ~ a v e c ~ u n e ~ a u t r e ~ s y l l a b a t i o n . ~ L e ~ L I V ², ~ p . ~ 276, ~}$ suggère néanmoins de rattacher à une même racine $* \mathrm{H}_{2} g$ - (sans représentant hors du

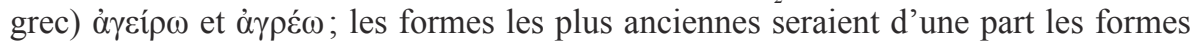

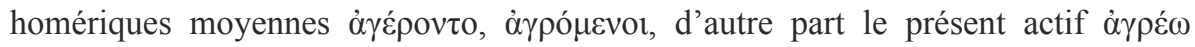
" prendre, saisir ", avec ses formes aspectuelles déjà employées en mycénien ( $a$-ke-

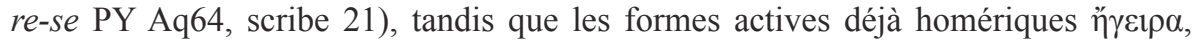
à $\gamma$ íp $\omega$ seraient secondaires. La présence par ailleurs dans une inscription de Datala,

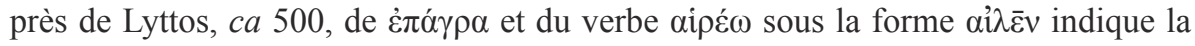
coexistence de ces deux radicaux dans le même dialecte, ce qui dissuade de faire l'hypothèse d'une origine commune de $\alpha \gamma \rho$ et $\alpha \iota \rho$, en considérant qu'ils se seraient simplement distingués comme variantes phonétiques et dialectales. V. Bernard-La Foucrière intervient en complément pour verser au dossier les données mycéniennes discutées par I. Hajnal, Mykenisches und homerisches Lexikon, 1998, p. 19, où myc.

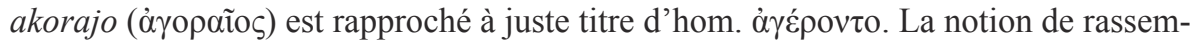
blement serait première ( $\alpha \gamma \varepsilon \rho-)$, d'où le développement de sens « collecter, saisir,

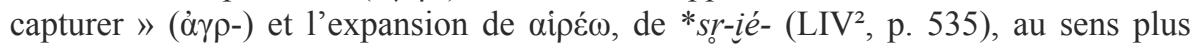

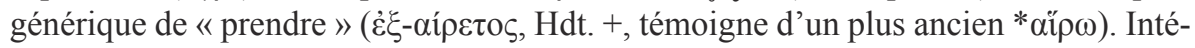
ressant développement de sens du nom d'agent autour de la notion de 'chef', déjà susceptible d'être véhiculée par la racine simple $* \mathrm{H}_{2}$ ger-. Comme ce développement concerne le radical $\alpha \gamma \rho-$, il faut considérer que la notion de rassemblement restait sous-jacente à celle de collecte et de saisie.

\section{Inscriptions dialectales du Péloponnèse et de Grèce septentrionale. Nouveautés dialectologiques}

Inscriptions d'Arcadie

- Mantinée :

$I G \mathrm{~V} 2262$ : jugement (début $\mathrm{V}^{\mathrm{e}} \mathrm{s}$.).

G.-J.-M.-J. Te Riele, « Hélisson entre en sympolitie avec Mantinée : une nouvelle inscription d'Arcadie », $B C H 111$ (1987), 167-190 (1 ${ }^{\text {er }}$ tiers IV ${ }^{\mathrm{e}}$ s.). Cf. L. Dubois, BCH 112 (1988), 167-190 et Thür-Taeuber, IPArk, n 9.

- Phénéos :

L. Dubois, RDA II, Phé1, 195-202 : loi sacrée archaïque.

- Cleitor : 
$R D A$ II, Cl.1, p. 208 : dédicace archaïque.

- Nouvelle inscription arcadienne apparue sur le marché des antiquités à Londres (origine présumée : Mont Lycée) : présentée par Laurent Dubois et Madeleine Jost en présence notamment de Jim Roy (Nottingham), spécialiste de l'Arcadie et de l'Élide et de J. Clackson (université de Cambridge, linguistique indo-européenne et grammaire comparée), co-auteur avec Y.-M. Carbon de la réédition de l'inscription dans Kernos 2016.

Le premier dialecte épigraphique d'étude est l'arcadien, avec pour commencer le jugement mantinéen pour impiété $I G \mathrm{~V} 2$ 262, daté par la forme des lettres du début $\mathrm{du} \mathrm{V}^{\mathrm{e}}$ siècle. Situation du dialecte et présentation des isoglosses avec le chypriote, $\mathrm{cf}$. e.g. les pages 417-426 de A. Panayotou, dans A.-F. Christidis, A History of Ancient Greek (publié en grec en 2001, traduit en anglais à Cambridge en 2007). Étude linguistique du texte et des anthroponymes mentionnés, comme Bó $\theta 1 \varsigma$, qui est loin d'être d'interprétation assurée, à cause notamment de l'ambiguité graphique avant l'époque de la réforme. Différentes interprétations possibles pour દ̇ $\sigma \kappa \lambda \alpha \rho \circ \zeta$, ligne 9, et la ligne

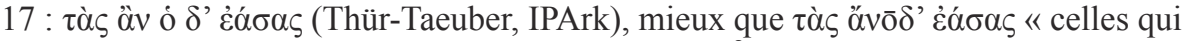
sont en haut » (Dubois), mais à éditer plutôt : $\tau \grave{\alpha} \varsigma$ àv $\hat{\delta} \delta \vec{\varepsilon}$ ' $\alpha$ ' $\sigma \alpha \varsigma$ (Strunk) « (et que des maisons soient partagées), celles dont il serait assurément propriétaire ». Graphie particulière ( $n u$ inversé) pour un phonème assez bien représenté à Mantinée avec cette même notation, qui a dû être transitoirement l'affriquée $/ \mathrm{t}^{\mathrm{s}} /$ issue de l'évolution de la labio-vélaire $* \mathrm{k}^{\mathrm{w}}$ devant voyelle prépalatale $e$ ou $i$, comme dans att. $\tau \varepsilon$ ou $\tau \iota \varsigma$; il évoluera ensuite pour rejoindre /t/, alors qu'un chypriote, c'est l'autre résultat possible qui a été conservé, d'où l'indéfini de forme ois dans ce dialecte. Problème de la lec-

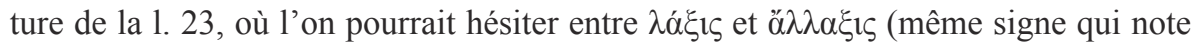
cette fois la séquence $/ \mathrm{ks} /$ ).

Traité de sympolitie entre la petite cité d'Hélisson et sa grande voisine, Mantinée.

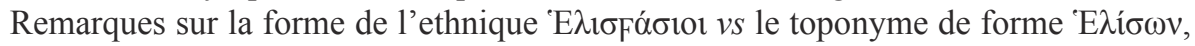

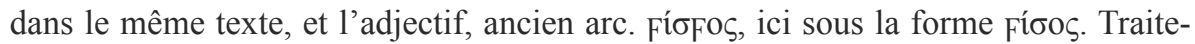
ment du groupe $-s w$ - issu de *tw. J'ai traité cette question dans la conférence en l'honneur d'Anne Jeffery à Oxford en juillet 2016, en proposant l'étymologie de la forme $\Gamma \varepsilon v \varepsilon ́ \sigma F \alpha v$ de la nouvelle inscription arcadienne (voir ci-dessous) : proposition étymologiques pour ce nom de fête et toponyme, s'inspirant aussi de l'article de L. Dubois des Mélanges offerts à Madeleine Jost, édités par P. Carlier et C. Cohen-Lerouge.

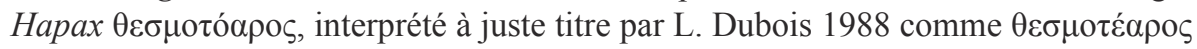

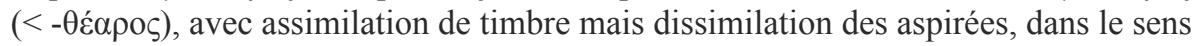
inverse de celui de la loi de Grassmann, donc secondaire et peut être isolée.

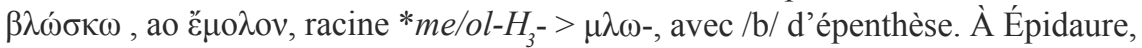

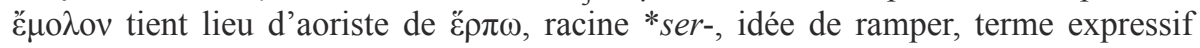
pour « marcher », certes, dans un des iamata, textes qui ne sont pas nécessairement exempts d'influence littéraire, ionienne en particulier (influence ds la littérature arétalogique). On a mlosk en tokharien A au sens de « s'enfuir », ce qui serait assez cohérent avec l'emploi comme supplétif de ع̌ $\rho \omega$, qui suppose un mouvement subreptice. Ce verbe est dialectal, dorien en particulier, y compris littéraire; il est bien ancré dans le Péloponnèse, et employé comme dialectal en poésie. Mais les deux thèmes se rencontrent dès Homère ( $\dot{\varepsilon} \kappa \mu \mathrm{\nu} \varepsilon \tilde{\varepsilon} v$ : Il. 11 604). Réflexions sur le statut qu'acquiert 
Hélisson par rapport à Mantinée qui l'englobe : la liberté ou autonomie cultuelle est conservée et les contrats de droit privé déjà passés restent inchangés; pour le reste, l'ancienne cité perd ce statut politique et acquiert celui de simple kôma de Mantinée, cf. Thür-Taeuber.

\section{Nouvelle inscription arcadienne}

Editio princeps (peu lisible) : J. Heinrichs, « Military integration in the late archaic Arkadia: new evidence from a bronze pinax (ca. $500 \mathrm{BC}$ ) of the Lykaion) », dans W. Heckel et al., The Many Faces of War in the Ancient World, Cambridge, 2015, 1-89; réédition (de référence) J.-M. Carbon, J. Clackson, «Arms and the boy. A New Sacrificial law of Ancient Arcadia », Kernos 29, 2016, 120-159.

Laurent Dubois précise ou amende la nouvelle édition du texte, dont il a donné une première notice dans le Bulletin épigraphique de la REG 2016, à partir de l'édition de travail diffusée par J. Clackson, qui avait fait circuler aussi les photos de la plaque de bronze (23 lignes lacunaires à gauche comme à droite, reconstituée à partir de plusieurs grands fragments jointifs) qu'il a pu autopsier. Des suggestions lors de cette séance vont permettre d'améliorer l'édition et d'élucider en particulier l'identification de certains toponymes ou noms de fêtes, sur lesquels les deux co-auteurs ont préféré avec prudence ne pas encore se prononcer. Saluons leur générosité intellectuelle, puisqu'ils ont donné dans des délais très brefs une édition de ce texte qui fera pour longtemps référence, en acceptant de laisser d'autres creuser un certain nombre de questions difficiles, qui méritent à elles seules d'autres études.

Il s'agit d'un calendrier qui réglemente des fêtes, les unes, annuelles, les autres triétériques, d'autres encore énétériques (les (H)oplodmia). Il semble que les fêtes prennent plus d'ampleur et durent alors trois jours tous les deux ans, et de même aussi tous les huit ans, tandis que les fêtes annuelles sont plus modestes et peut-être, en dépit des éditeurs, pourraient ne durer qu'une journée (si l'on avalisait le $\mu$ tóv $\beta \rho 1$ qu'ils corrigent $<\tau \rho>1 \alpha ́ v \beta \rho \imath, 1.1)$. A noter la glose d'Hésychius $\alpha \mu \beta \rho i ́ \zeta \varepsilon ı v \cdot \theta \varepsilon \rho \alpha \pi \varepsilon v ́ \varepsilon ı v$

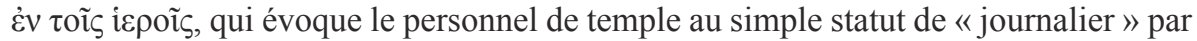
opposition aux magistrats prêtres. Il est vrai que n'est jusqu'ici attesté aucun composé à premier élément $\mu \mathrm{t}$, susceptible d'avoir été fait à partir du radical d'un adverbe multiplicatif** $\mu^{*} \zeta$, dont on pourrait conjecturer qu'il soit analogique de $\delta i ́ \zeta$ et de $\tau \rho i ́ \varsigma$. Il serait peut-être moins difficile de partir du radical du numéral au féminin $\mu$ í $\alpha$ dont

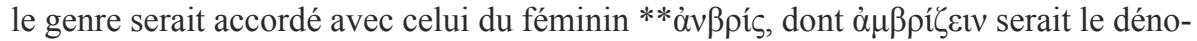
minatif. Cela supposerait d'y reconnaître un substantif plutôt qu'un adjectif substantivé, un dérivé du neutre ท̃ $\mu \alpha \rho$. Plutôt qu'un composé, on aurait alors un juxtaposé, du

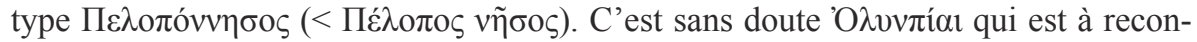

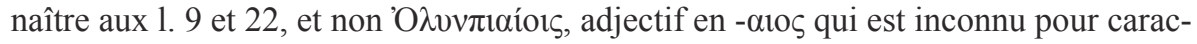
tériser tant les concours olympiques (O $\lambda v v \pi \imath \varepsilon \tilde{\imath} \alpha)$, que le mois où ils avaient lieu ou le Zeus d'Olympie ('O

Une séance supplémentaire a été consacrée à l'inscription : nouvelles propositions

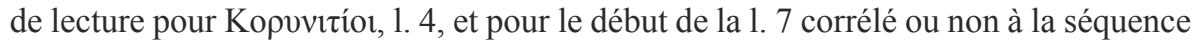

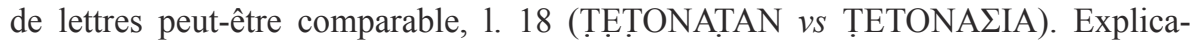
tion de la forme $\Gamma \varepsilon v \varepsilon ́ \sigma F \alpha$ attestée à trois reprises, 1.14 et 18. Réflexions sur TOTINIOI, 1. 7. Questions de la datation, à partir de l'examen de la forme des lettres, et de 
l'origine géographique, à partir de la comparaison des procédés graphiques employés dans les différentes cités arcadiennes pour noter l'affriquée sourde $\left[\mathrm{t}^{\mathrm{s}}\right]$.

Présentation ensuite de la loi sacrée arcadienne de Phénéos, concernant le culte à Déméter Thesmophoros, datée de ca 500 a.C., Dubois, RDA II, 195-208 : inter-

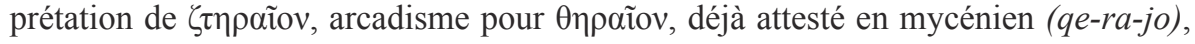
en référence soit à Théra, et à des tissus à la façon ou à la couleur caractéristique de l'île, soit aux peaux de bêtes, l'interdit cultuel pouvant s'expliquer par la fonction de législatrice de Déméter, susceptible de faire passer de la barbarie à l'état de civilisation, cf. M. Jost, Sanctuaires et cultes..., 323-324. Emploi unique du verbe õ $\sigma \alpha 1$, ici préverbé en $\alpha \dot{\pi} 0-([\dot{\alpha} \varphi \alpha ́ \varepsilon] \sigma \tau \alpha 1, \alpha \dot{\alpha} \varphi \alpha ́ \varepsilon \tau o 1)$, au sens, non de "rassasier, dégoûter » comme le simple actif, mais dans ce contexte juridique, au moyen, de « se délester, dégraisser » : ce dernier sens, à l'acception financière de « débarrasser d'un excédent de biens ", comme on l'emploie à propos d'un budget, est attesté en français fami-

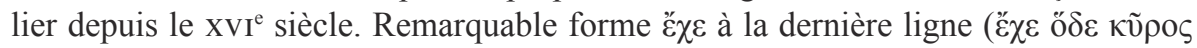

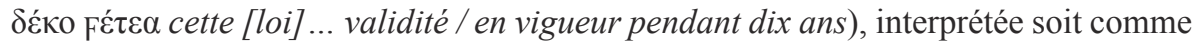
un subjonctif présent (en arcadien, sans iota analogique) d'ordre, emploi certes peu canonique, soit comme une forme ancienne d'injonctif sans désinence étendue à la $3^{\mathrm{e}}$ pers. du sg. Il pourrait s'agir aussi, à condition de corriger $\varepsilon \chi_{\varepsilon}<1>$, d'un indicatif présent, faisant remonter la validité de la loi à l'instant où elle a été votée.

Autres nouveautés dialectologiques : inscriptions de Thessalie, à partir du corpus des Inscriptions d'Atrax publié en 2016 par R. Bouchon, B. Helly et L. Darmezin.

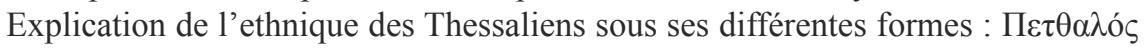

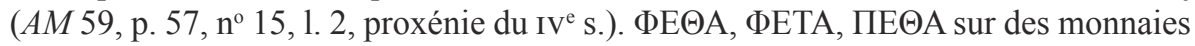
d'argent $d u v^{e}$ s., à côté de la forme adoptée à l'extérieur $\Theta \varepsilon \sigma \sigma-$, atticisée en $\Theta \varepsilon \tau \tau-$ ou béotisée en $\Phi \varepsilon \tau \tau-$ (cf. M. H. Hansen, T. H. Nielsen, An Inventory of archaic and classical poleis, Oxford 2004, notice « Thessalie » de J.-C. Decourt, T. H. Nielsen et

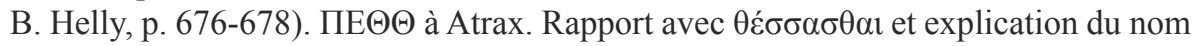
A $\gamma \lambda \omega-\theta \varepsilon \dot{\sigma} \sigma \eta \varsigma$ (Amorgos, IV s.).

Étude de l'inscription $\mathrm{n}^{\circ} 1$, un jugement de droit public intérieur à la cité d'Atrax, daté sur la forme des lettres, proche de celles de la stèle des Basaidai (B. Helly, $B C H$ 94, 161-189), de l'époque hellénistique.

Analyse du système de dénomination patronymique des Thessaliens à partir de l'étude de la liste des noms des juges et des témoins. Adjectifs patronymiques en $-\varepsilon 10 \varsigma,-\alpha 10 \varsigma$, cf. l'article de B. Helly, R. Bouchon dans La suffixation des anthroponymes grecs antiques, Genève, 2017, 559-578.

Désinence $-\alpha \varepsilon v$ d'indicatif aoriste $3^{\mathrm{e}} \mathrm{pl}$. (et variantes - $\alpha \iota \varepsilon v, \alpha \iota v$ ) et ses liens avec la

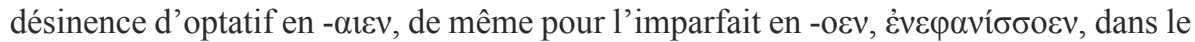
décret de Larissa accompagnant la lettre de Philippe V de Macédoine aux Larisséens datée de 217/216 a.C., IG IX 2, 517, 1. 3. Une forme d'imparfait $3^{\mathrm{e}} \mathrm{sg}$. cí a été récemment étudiée par L. Dubois, dans Mélanges en l'honneur de F. Bader, 2012, "Thessalika », 45-55 : elle côtoie $\varepsilon \tilde{i} \zeta$, équivalent de dor. $\tilde{\eta} \varsigma$, forme de prétérit munie de désinences actives correspondant au présent غ̇ $\sigma \tau i$, lat. est. La coexistence de formes de prétérit $3^{\mathrm{e}} \mathrm{sg}$. et pl. à l'allure d'optatif et l'emploi de certaines d'entre elles dans des complétives où l'on aurait en prose attique classique des optatifs obliques, invite à postuler, à la suite déjà d'A. Morpurgo Davies (Glotta 43, 1965, p. 235-251), une 
convergence qui s'explique par l'ancrage sémantique commun dans la sphère du non actuel.

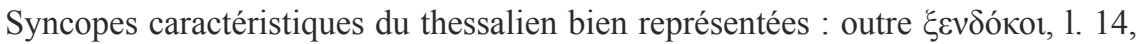

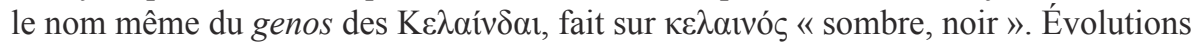
phonétiques notables : Bov- $\lambda \varepsilon \pi \alpha \rho i ́ \delta \alpha 1$, avec /i/ > /e/ au voisinage de la liquide /1/;

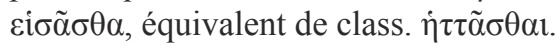

Étymologie d'anthroponymes et de phylonymes rares ou nouveaux : Av̉ $\rho 0 \gamma i ́ o v v \delta \alpha 1$,

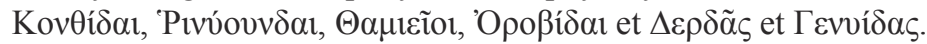

Étude du décret d'une cité éolienne non identifiable en l'honneur des Atragiens qui l'avaient approvisionnée en blé lors d'une disette, probablement au milieu du $\mathrm{II}^{\mathrm{e}} \mathrm{s} . a . C$.

Présentation des traits dialectaux et des éléments du contexte qui parlent en faveur d'une cité d'Éolide d'Asie Mineure et non de Grèce propre : emploi notam-

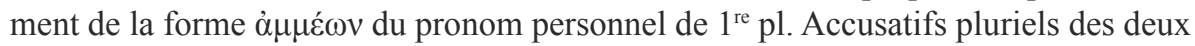
premières déclinaisons systématiquement en -aıs et -ots, participe au nom. masc.

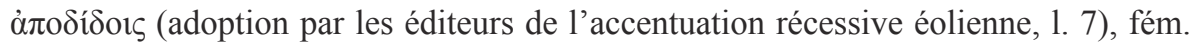

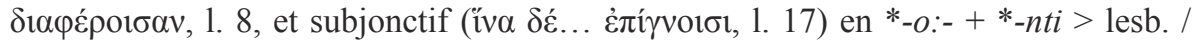

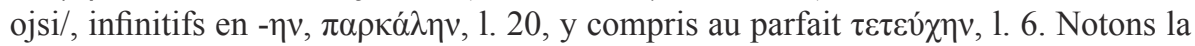

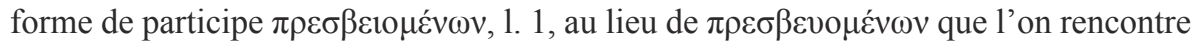
partout ailleurs, dont la diphtongue médiane ne peut être qu'analogique. Le composé

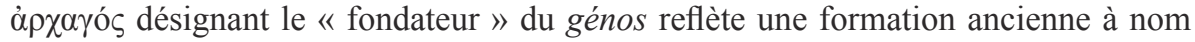
d'agent thématique au second élément, avec son accent hérité sur la voyelle thématique, cf. en dernier lieu D. Petit, « Force et dominance accentuelle en morphologie dérivationnelle grecque », dans A. Blanc, D. Petit (éd.), Nouveaux acquis sur la formation des noms en grec ancien, Paris, 2016, 21-24. La réfection en à $\rho\rceil \gamma \varepsilon \dot{\varepsilon}-\tau \eta \varsigma$ a permis de doter le composé d'un suffixe formateur des seuls noms d'agents, quand la voyelle thématique, morphologiquement polyvalente, rendait l'interprétation ambiguë. Les deux lexèmes sont attestés aussi anciennement, mais sont l'un et l'autre postérieurs à l'épopée.

Le 21 mars, une conférence de Catherine Dobias-Lalou, professeur émérite à l'université de Bourgogne, a réuni comme objet d'étude un dossier de souscriptions et de comptes de démiurges de Cyrène, des périodes classique et hellénistique. Le sujet était celui des systèmes de numération qui se succèdent à Cyrène. Le plus ancien est lié aux unités monétaires, avec des signes parfois acrophoniques, comme $\mathrm{X}$ pour les milliers ( $\chi \eta \dot{\lambda} \lambda$ เo en dorien local) de mines et de dichalques, $>$ qui note 10 dans les mêmes unités monétaires, et pourrait correspondre au L orienté différemment de la stèle des Géléontes publiée par D. Rousset (Journal des savants 2014, 32 ), et interprété par lui comme valant une demi-drachme. Se pratique par ailleurs la notation alphanumérique, avec notamment l'emploi de sampi $(\mathbb{K})$ aussi parakuïsma pour référer aux milliers : seul, il signifie « mille », diacrité à l'aide de petits $\mathrm{A}, \mathrm{B}, \Gamma$, etc. qui le surmontent, il indique leurs multiples. Les corpus en ligne des inscriptions grecques de Cyrène, IGCyr, et GVCyr, celui des inscriptions métriques, tous deux publiés sous la direction de notre invitée et hébergés à l'université de Bologne, sont consultables à l'adresse https://igcyr.unibo.it. 
Poursuite de notre étude des inscriptions d'Atrax par celle de la dédicace $\mathrm{n}^{\circ} 51$, à Apollon, d'Euphorbe l'archi-dauchnaphore et son collège de syndaphnaphores

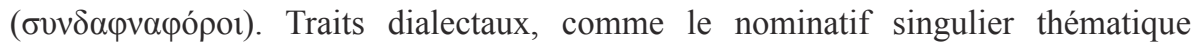

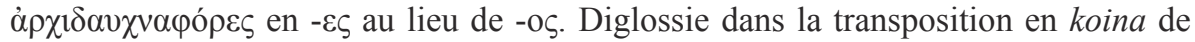
cette désignation d'une fonction cultuelle, qui s'explique sans doute moins par la nécessité de traduire que par la volonté de manifester son identité dialectale tout en rendant manifeste sa maîtrise de la langue de prestige. Étymologie du nom du laurier

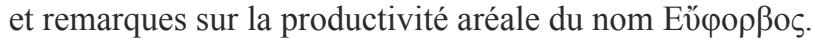

Quatre conférences sur Dialectes grecs et histoire de la langue du professeur J. Rau, université Harvard, directeur d'études invité communément avec G.-J. Pinault, de fin avril à fin mai 2017. Successivement : 1. Les dialectes grecs : filiation, diffusion et convergence. 2. Les dialectes grecs au $\mathrm{II}^{\mathrm{e}}$ millénaire : phonologie et répartition en sous-groupes. 3. Les dialectes grecs au $\mathrm{II}^{\mathrm{e}}$ millénaire : morphologie et répartition en sous-groupes. 4. Archaïsme, innovation et dialectologie dans la langue homérique. À partir de l'établissement d'une chronologie relative et de la datation absolue d'innovations, confirmation de la division traditionnelle des dialectes en quatre groupes : achéen (arcadien, chypriote et mycénien), ionien-attique, éolien (lesbien, thessalien et béotien) et grec occidental (parlers du Nord-Ouest, dorien dit « sévère », etc.). Tentative de reconstruction de la proto-histoire linguistique du monde grec, à partir de l'étude comparée minutieuse de traits phonétiques et morphologiques des dialectes du second et du premier millénaire. Catégorisation des différents modes d'évolutions : la notion néogrammairienne d' " héritage », partielle, comme l'avait remarqué Brixhe (dans Peuplements et genèses dialectales dans la Grèce antique, 2006), est complétée à l'aide de celles de « diffusion » et de convergence (« when dialects or distinct languages are in contact and linguistics innovations spread or are diffused from one linguistic group to another »), voir aussi ma contribution sur le sujet dans l'Encyclopedia of Ancient Greek Language and Linguistics, éd. G. K. Giannakis, 2014, vol. 1, s.v., 454-457. Reconstitution de l'histoire de l'évolution de l'emploi de digamma et des différentes formes de la désinence de génitif singulier thématique (att. -ov) dans la langue homérique et à travers les interventions éditoriales de ses commentateurs antiques (Aiveí $\omega<0>$ chez Zénodote, Il. 5, 263 et 323).

Conclusion de nos travaux thessaliens par l'étude d'un décret hellénistique trouvé à Aigai d'Éolide et émanant des Thessaliens, offrant atéleia, droit de cité et epigamia à des citoyens d'Éolide, de Cos et de Magnésie du Méandre (H. Malay et M. Ricl, Epigraphica Anatolica 42 (2009), 39-60, cf. B. Helly, Bull. 2010, n 522, 832-836; R. Parker ZPE 177 (2011), 111-118; SEG 2009 [2012], n 1406B). Nom du prêtre de forme $\lambda \varepsilon ı$ ó $\rho \alpha \varsigma, 1$. 11, mis en relation avec des noms propres faits à partir du même radical par O. Masson, RPhi 37 (1963), 216-217, notamment A’ Des gloses d'Hésychius font mettre en relation formes avec et sans « prothèse ». Un article de G. Genevrois, RPhi 89 (2015) [2017], 73-108, s'intéresse précisément à ces gloses. Le dossier étymologique de ce nom de prêtre et des anthroponymes cor-

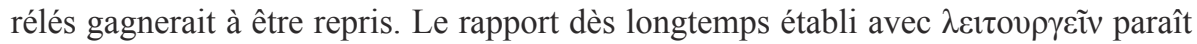
en effet devoir être écarté, comme le signalait déjà Chantraine, $D E L G$, s.v. $\lambda \eta ́ \tau \omega \rho$ : 
$\lambda \eta ́$ itov « maison commune » appartient à la famille étymologique de $\lambda \bar{\alpha}(\mathrm{F})$ ó $\zeta$, cf. myc. $r a$-wa-ke-ta (alph. $\lambda \bar{\alpha} \gamma \dot{\varepsilon} \tau \bar{\alpha} \varsigma$ ), et le /a:/ d'origine paraît incompatible avec le /e:/ systématiquement attesté au radical du nom du prêtre, qu'il prenne la forme $\lambda \varepsilon \imath \tau-, \lambda \eta \tau-$

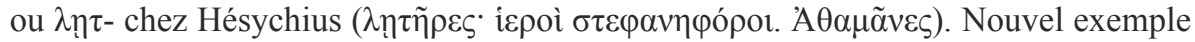
de l'imparfait de concordance dans le discours rapporté au passé, eľ́otev, 1. 12-13, à désinence thématique non -ov, mais $-0-1 \varepsilon-v$. Apports étymologiques récents sur le nom d'Héra et du héros, cf. G.-J. Pinault, « The Greek Hero: the Man with Strong Bones », dans Essays for the centenary of Georgi Mihailov (1915-1991), 2016, 478487 (*hich $\left.h_{1} r o-h_{2} s t-\right)$, avec au premier élément la racine de $̋ \rho \alpha$, et J. L. Garcia Ramon "Hera and Hero: Reconstructing Lexicon and God names », dans D. Goldstein, S. Jamison, B. Vine (éd.), Proceedings of the 27th Annual UCLA Indo-European conference, $2016\left(* h_{l}\right.$ ieh $\left.{ }_{1}{ }^{-o u-}\right)$ : même proposition étymologique pour le radical, même suffixation que $\pi \alpha ́ \tau \rho \omega \varsigma, \mu \eta ́ \tau \rho \omega \varsigma$, et réfections intraparadigmatiques qui expliqueraient l'absence de /w/ dès le myc. ti-ri-se-ro-e, datif du juxtaposé formé sur $\tau \rho i ́ \varsigma$ $+\eta ๊ \rho \omega \varsigma$ « Triple-héros ». G. J. Pinault n'avait pas eu connaissance, pour sa part, des formes en argien de Cléonée, dans une inscription du $\mathrm{v}^{\mathrm{e}}$ s. a.C. qui doit être publiée

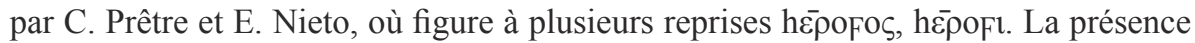
de /w/ en dorien du premier millénaire, alors qu'il paraît absent au second, fait problème. Il est difficile de suivre, pour cette haute période, l'hypothèse de l'hyperdialectalisme proposée, faute de mieux, par J. L. Garcia Ramon . Il serait préférable de raisonner en termes de variations phonétiques. Même s'il nous manque pour l'instant une forme mycénienne en e-ro-w\#, il pourrait être utile de la postuler, à côté de la seule qui nous soit pour l'instant connue : ce maillon manquant ferait mieux comprendre ces formes doriennes à /w/ intervocalique, même s'il est vrai que la séparation majeure établie dans la proto-histoire des dialectes entre grec méridional (dont relève le mycénien) et septentrional (éolien et différentes variétés de doriens) pourrait à elle seule justifier l'importante différence de chronologie dans l'amuïssement de /w/ hérité. 